A. Weisbecker, M. Burmester \& A. Schmidt (Hrsg.): Mensch und Computer 2015 Workshopband, Stuttgart: Oldenbourg Wissenschaftsverlag, 2015, S. 11-18.

\title{
Nutzeranforderungen eines Systems zur automatischen Helferbereitstellung
}

\author{
Henrik Detjen, Stefan Geisler, Gerd Bumiller
}

Institut für Informatik, Hochschule Ruhr-West

\section{Zusammenfassung}

Bei Großschadensereignissen kann es durch die Vielzahl der Alarme dazu kommen, dass die verfügbaren Rettungskräfte nicht mehr ausreichen, um die anfallenden Aufgaben zu bewältigen oder Hilfsfristen einzuhalten. Die vorliegende Arbeit beschreibt einen Ansatz, sich zusätzlicher Hilfe aus der Bevölkerung zu bedienen, die über einen Disponenten aus der vorhandenen Leitstelle koordiniert wird. Dabei stehen nicht spontan organisierte Helfer im Vordergrund, sondern Personen, die sich vorab mit einem klaren Fertigkeitsprofil und ggf. auch Ausstattung im System registriert haben. Besondere Anforderungen entstehen bei den Disponenten der Leitstelle, deren Mehrbelastung durch das neue System gering zu halten ist, als auch bei den freiwilligen Helfern, die über eine App auf dem Mobiltelefon alarmiert werden und auch darüber die Kommunikation führen sollen. Die Anforderungen beeinflussen sowohl die System-Infrastruktur als auch die Benutzerschnittstelle.

\section{$1 \quad$ Einleitung}

Das hier vorgestellte Projekt „Automatisiertes Helferangebot bei Großschadensereignissen“ (AHA) wird in Kooperation der Hochschule Ruhr-West, der Fachhochschule für öffentliche Verwaltung Duisburg, der Universität Duisburg-Essen, der Firma CKS Systeme GmbH und des Instituts für Rettungstechnik (IFR) Dortmund durchgeführt.

Durch Analysen vergangener Großschadensereignisse in Vorbereitung dieses Projektes wurde festgestellt, dass die Ressourcen der Rettungskräfte in besonderen Fällen nicht mehr ausreichen, um die Anzahl der anfallenden Rettungs- und Sicherungsaufgaben in angemessener Zeit zu erledigen. Es kann überregional Hilfe angefordert werden, doch auch diese steht bei Großschadenslagen häufig nicht zur Verfügung. Das Ziel des Projektes ist es zusätzliches, qualifiziertes Potential aus der Bevölkerung zu mobilisieren und den Disponenten nutzbar zu machen. Zusätzliche Helfer aus der Region und technische Hilfsmittel werden zu diesem Zweck erfasst, überprüft und registriert, sodass im Notfall darauf zurückgegriffen werden kann. Über eine mobile App können Standort und Verfügbarkeit ermittelt werden 
und somit als Entscheidungskriterium verwendet werden. Die Entscheidung über den Einsatz der Helfer selbst erfolgt nicht automatisiert, sondern durch den Disponenten.

Durch die Möglichkeit, im Fall von Großschadensereignissen schnell zusätzliche, qualifizierte Ressourcen zur Verfügung zu stellen, soll AHA zu einer wesentlichen Verkürzung der Hilfsfrist beitragen. Dabei ist die Realisierung niedriger Investitions- und Betriebskosten ein zentrales Ziel, ebenso wie die Einhaltung der Persönlichkeitsrechte der freiwilligen Helfer.

Im Verlauf werden zunächst einige verwandte Projekte (Kapitel 2) vorgestellt und das AHAProjekt in diesem Kontext abgegrenzt. Darauf folgt ein Überblick über das Systemkonzept (Kapitel 3) und mögliche Einsatzszenarien (Kapitel 4). Zuletzt werden konzeptionelle Herausforderungen beschrieben (Kapitel 5) und ein kurzer Ausblick gegeben (Kapitel 6).

\section{Verwandte Projekte}

Vor dem Hintergrund der Jahrhundertflut im Sommer 2013 entstand das Projekt „Hands2Help“ (Hofmann et al. 2014). Ziel ist es hier die eher unkoordinierte freiwillige Hilfe durch eine mobile App zu organisieren. Einer virtuellen Pinnwand gleich, können Aufgaben ins System eingetragen werden und von (ad-hoc) registrierten Helfern übernommen werden. Ein Vermittlungsalgorithmus berücksichtigt dabei die Nähe zum Einsatzort per GPS und die Eignung für den Einsatz, z.B. benötigte Hilfsmittel wie Schaufeln. So sollen Hilfswillige an den richtigen Ort zur richtigen Zeit vermittelt werden, um die Effizienz der Hilfe insgesamt zu erhöhen. Außerdem bestehen Schnittstellen für Leitstellen, die es ermöglichen mit den Helfern vor Ort zu kommunizieren und diesen weitere Informationen zu ihrem Einsatz zukommen zu lassen oder Informationen abzufragen.

Das Projekt „United Hatzalah“ ${ }^{1}$ (Chan et al. 2007) unterstützt medizinische Einsatzkräfte bei lebensrettenden Maßnahmen. Mit mehr als 2500 Helfern ist es die größte, unabhängige Freiwilligenorganisation Israels in diesem Bereich. Der Kern ist eine mobile Anwendung mit dem sogenannten „LifeCompass system“: Im System eingehende Notfälle werden durch den Einsatz von GPS-Technologie an in der Nähe befindliche Helfer weitergeleitet. Davon rückt dann der Qualifizierteste aus. Es haben sich im Laufe der Zeit sogar eigene kleine Leitstellen entwickelt, die die Freiwilligen und deren Hilfsmittel koordinieren. Zu den Hilfsmitteln zählen Defibrillatoren, Motorroller mit medizinischer Ausrüstung, aber auch komplett ausgestatte Krankenwagen. Die durchschnittliche Hilfsfrist liegt nach Eigenangaben bei 3,5 Minuten.

Ein ähnliches Projekt gibt es in Deutschland unter dem Namen „Mobile Retter“ ${ }^{2}$. Hier werden ebenfalls medizinische Notfälle durch freiwillige Helfer unterstützt. Sobald ein Notfall im System eingeht, werden die im System erfassten Helfer in der Nähe kontaktiert und her-

1 https://israelrescue.org/about-us.php

2 http://www.mobile-retter.de/ 
angezogen. Das Projekt ist 2013 in die Probephase gegangen und derzeit sind bereits über 400 Freiwillige registriert. Mit der Anmeldung bekommt der Helfer einen Termin für ein Seminar beim Mobile Retter e.V., im Zuge dessen eine Ausbildung für die Nutzung des Systems durchgeführt wird.

Verschiedene andere Projekte und spontane Aktionen verfolgen einen kollaborativen Ansatz, teils über soziale Netzwerke. Hierbei ist es jedoch schwierig, einen Gesamteindruck der Situation zu erhalten und die verfügbaren Kräfte optimal einzusetzen, so dass viel Potenzial ungenutzt bleibt bzw. an anderen Orten Fähigkeiten fehlen. Ein umfassender Überblick zu dieser Thematik wird in (Reuter et al. 2014) sowie zu den Herausforderungen in (Kaufhold \& Reuter 2014) gegeben.

In den eben beschriebenen Ansätzen lassen sich zwei übergeordnete Motive ausmachen:

- Die Verkürzung der Hilfsfrist im medizinischen Notfall.

- Die Entlastung der Rettungskräfte beim Großschadensereignis.

Die Ziele des AHA-Projektes sind denen der genannten Projekte ähnlich, eine Verkürzung der Hilfsfrist, aber nicht nur im medizinischen Bereich. Jedoch soll die Hilfe besser koordiniert werden, als in reinen Community-basierten Ansätzen, damit das Hilfspotenzial zielgerichtet eingesetzt werden kann. Hierzu sollen die üblichen Leitstellen, etwa der Feuerwehren, die zentrale Koordination auch für die freiwilligen Helfer übernehmen. Daher ist das Ziel von AHA die Integration in bestehende Systeme und Prozesse. Dabei ist zu untersuchen, wie über einen längeren Zeitraum freiwillige Personen zur Teilnahme motiviert werden können, wie deren Erreichbarkeit unter Beachtung von Datenschutzanforderungen zu gewährleisten ist und wie die Disponenten diese zusätzlichen Ressourcen in Stresssituationen mit möglichst geringerer zusätzlicher Belastung einbeziehen können. Nicht im Fokus des Projektes sind sich spontan bildende Helfergruppen, sondern ausschließlich vorregistrierte Personen.

\section{Ansatz}

Die Umsetzung des AHA-Projekts wird auf zwei Seiten erfolgen. Zum einen muss ein System auf Seite der Disponenten in den Leitstellen entwickelt werden, welches Informationen über zusätzliche Helfer empfängt, verarbeitet und weiterleitet. Dieses muss entsprechende Schnittstellen zur Integration in die Leitstellensoftware bereitstellen. Zum anderen muss ein System für die Helfer entwickelt werden, das Informationen an die Leitstellen übermittelt. Hierfür eignet sich, aufgrund der hohen Verbreitung und der potentiell hohen Erreichbarkeit, der Einsatz auf mobilen Endgeräten. 


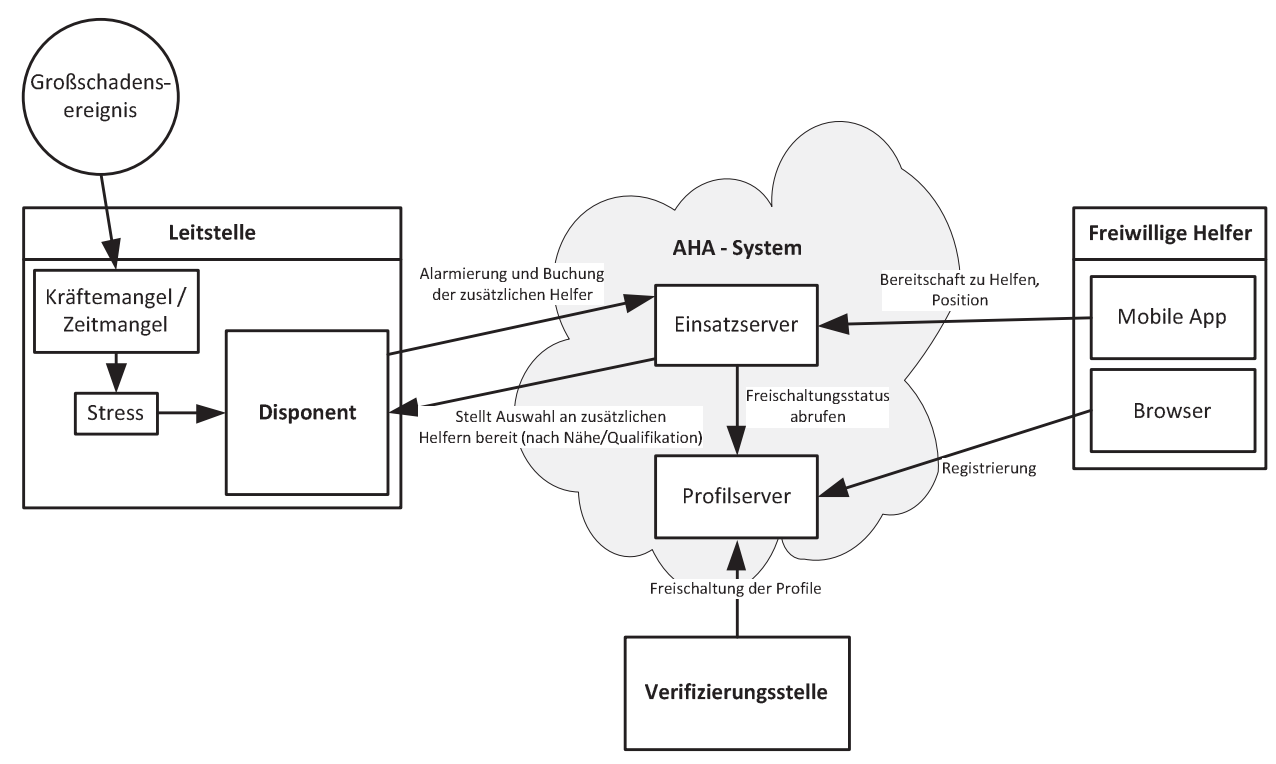

Abbildung 1: Überblicksskizze AHA-Projekt

Abbildung 1 zeigt überblicksartig eine Entwurfsskizze: Bei einem Großschadensereignis stehen in der Leitstelle oft nicht genug Kräfte zur Verfügung und es tauchen gleichzeitig viele kritische Einsätze auf. Dies hat zur Folge, dass die Disponenten unter einem besonders hohen Druck stehen. Das AHA-System soll den Disponenten entlasten und zusätzliche Kräfte in Form von freiwilligen Helfern bereitstellen. Diese sollen sich im Vorhinein über eine Webseite registrieren. Dabei werden persönliche Daten, Qualifikationen, sowie mögliche Hilfsmittel erfasst. Eine Verifizierungsstelle prüft die Angaben der Helfer auf Richtigkeit. Details zur Verifizierungsstelle werden im weiteren Verlauf des Projektes erarbeitet: Dazu gehören neben technischen Aspekten auch rechtliche, organisatorische und finanzielle Rahmenbedingungen. Es ist ebenfalls darauf zu achten, dass keine Motivationshürde bei potenziellen freiwilligen Helfern aufgebaut wird.

Durch die Trennung von Profil- und Einsatzserver wird die Sicherheit der persönlichen Daten erhöht, denn auf dem Einsatzserver befinden sich nur die für einen Einsatz relevanten Daten: Qualifikation und Hilfsmittel - gekoppelt an ein Pseudonym. Kommt es zu einem Einsatz, kann der Disponent in seinem Leistellensystem die in der Nähe befindlichen und für den Einsatz geeigneten Helfer einsehen, alarmieren und den Geeignetsten auswählen.

Der Bereitschaftsstatus eines Helfers muss ,,verfügbar“" sein, damit er im System berücksichtigt wird. Kommt ein Einsatz beim Disponenten an, werden erste Anfragen im Hintergrund an die Mobilgeräte der Helfer gesendet und deren Positionen ermittelt. Diejenigen, die sich in Reichweite zum Einsatzort befinden, werden dem Disponenten dann vorgeschlagen. Wählt dieser einen Helfer aus, bekommt er eine Push-Nachricht auf sein Mobilgerät. Darin wird der Einsatz abstrakt beschrieben (Art und Nähe). Bei Annahme des Einsatzes, werden 
genauere Information bereitgestellt und eine Route zum Einsatzort vorgeschlagen. Zur Zeit sind noch alternative Alarmierungskonzepte in Arbeit, wie z.B. eine an Einsatzlagen oder Auslastung gekoppelte Alarmierung, bei der ein Helfer seinen tatsächlichen Bereitschaftsstatus ab einer bestimmten Auslastung erneut bestätigen muss. Der Trade-Off besteht hierbei zwischen Unsicherheit beim Disponenten (Ablehnungsquote) und Toleranz beim Nutzer (Anzahl der Bereitschaftsabfragen). Hier sollen unterschiedliche Konzepte getestet werden.

\section{Szenarien}

Das IFR hat in Zusammenarbeit mit der Feuerwehr Dortmund zwei mögliche Einsatzszenarien für das System definiert. Diese werden im Folgenden verkürzt dargestellt.

\section{Szenario Großschadensereignis:}

In Dortmund wütet über Tage ein Unwetter, vergleichbar Kyrill 2007. Es kommt in sehr kurzer Zeit zu fast 2000 Notrufen. In einem Industriegebiet, das an ein Wohngebiet angrenzt, brennt nach einem Blitzeinschlag eine Werkhalle. Das Feuer breitet sich schnell aus und wird durch Windböen angefacht. Gleichzeitig kommt es auf einer Bundesstraße zu einem Autounfall mit Verletzten, die von einem Baum eingeklemmt werden. Durch diese beiden Ereignisse sind alle Mitarbeiter und Fahrzeuge der Feuerwehr, des THWs und des Rettungsdienstes im Einsatz.

Fast zur gleichen Zeit gehen mehrere Notrufe von Anwohnern ein, deren Zufahrtsweg durch einen umgefallen Baum blockiert wird. Im AHA-System sind Personen registriert, die bereit sind ihre stark motorisierten Fahrzeuge, z.B. Traktoren, der Feuerwehr leihweise bereit zu stellen. Diese Maschinen werden den Disponenten als verfügbare Ressourcen vorgeschlagen und können beim Besitzer anfragt werden. Mit Hilfe einer bereitgestellten Maschine kann die Feuerwehr den Baum zeitnah von der Straße räumen.

In der Innenstadt sind durch Starkregen mehrere Keller vollgelaufen, entsprechende Notrufe gehen ein. Das AHA-System schlägt mehrere Tauchpumpen vor, bei deren Auswahl die Besitzer alarmiert werden, um diese zum Einsatzort zu bringen.

Zusätzlich sind im AHA-System mehrere „Erkunder“ registriert, welche bei nichtmedizinischen Notfällen aktiv werden. Sie fahren Notrufe ab, bei denen es sich um umgefallene Bäume oder ähnliches handelt und teilen der Leitstelle mit, ob ein Eingreifen der Feuerwehr weiterhin notwendig ist. Wenn z.B. durch nachbarschaftliche Hilfe ein Baum bereits geräumt wurde, können so Leerfahrten der Einsatzkräfte vermieden werden.

\section{Szenario Medizinischer Notfall:}

Im Autobahnkreuz Dortmund-Mengede, einer Schnittstelle zwischen A2 und A45, kommt es zu einem Verkehrsunfall zwischen einem vollbesetzten Reisebus und einem Gefahrenguttransporter. Es gibt über 50 Verletzte und es läuft ein Einsatz zur ABC-Gefahrenabwehr an. In sehr kurzer Zeit sind große Teile des Dortmunder Rettungsdienstes im Einsatz. In das Einsatzkonzept sind auch Einheiten der Freiwilligen Feuerwehr, für die medizinische Erst- 
versorgung, und Rettungsdienste umliegender Regionen eingebunden. Für weite Teile des Stadtgebiets ist es absehbar, dass eingehende Notfälle nicht in der Hilfsfrist von 8 Minuten erreicht werden können. In der Leitstelle der Feuerwehr Dortmund gehen täglich 300 Notfälle ein, von denen ca. 20\% einen Notarzt-Einsatz begründen. Paralleleinsätze zum Autobahneinsatz sind zu erwarten.

Bei einem Notruf aus dem Ortsteil Höchsten wird gemeldet, dass ein Familienangehöriger mit einem Herz-Kreislauf-Stillstand zusammengebrochen ist. Der nächste Rettungswagen aus der Stadtmitte hat eine Anfahrtszeit von ca. 19 Minuten. Im AHA-System sind der Feuerwehrmann X, der nach Alarmierung seines Löschzuges nicht rechtzeitig ausrücken konnte, und die Intensivpflegerin Y, die Urlaub hat und bei der Gartenarbeit ist, registriert. Beide sind nur wenige hundert Meter entfernt von der Notrufadresse und werden dem Disponenten vom AHA-System zusätzlich zum Rettungswagen und Notarzt vorgeschlagen. Dieser alarmiert beide zusätzlichen Helfer, welche dann drei Minuten später am Unfallort eintreffen und mit Wiederbelebungsmaßnahmen beginnen. Nach weiteren 15 Minuten wird der Patient mit regelmäßigem Eigenpuls dem Notarzt übergeben.

\section{Herausforderungen}

In diesem Kapitel werden auszugsweise die bei der Konzeption aufgetretenen Herausforderungen verschiedener Projektbereiche geschildert und diskutiert.

Rechtssicherheit: Da das AHA-System von der Feuerwehr, also einer staatlichen Organisation, einen Auftrag an den Bürger sendet, gilt es hier zu untersuchen, wie das Haftungsverhältnis zwischen den beiden Parteien im Einsatzfall ausgestaltet ist.

Persönlichkeitsrechte: Bei der Registrierung und im Einsatzbetrieb müssen stets die Persönlichkeitsrechte der freiwilligen Helfer gewahrt werden. Die konzeptionelle Trennung von Profil- und Einsatzserver garantiert, dass der Disponent keinen Zugriff auf persönliche Daten des Helfers erhält, sondern nur auf Grundlage von Qualifikation und Einsatznähe entscheidet. Alle Verbindungen sollen nach aktuellen Standards verschlüsselt werden.

Technische Herausforderungen: Neben der Zuverlässigkeit des Systems ist vor allem die Genauigkeit der GPS-Lokalisierung für dessen Erfolg verantwortlich. Positionswerte müssen präzise von der Leitstelle ermittelt werden können. Dabei muss untersucht werden, inwiefern die Genauigkeit und Aktualität der Ortung den Energieverbrauch der mobilen App erhöhen.

Nutzungsmotivation: Um das Ziel einer langfristigen Nutzung durch sowohl Disponenten als auch freiwillige Helfer zu erreichen, soll untersucht werden, welche Faktoren den Helfer dazu bewegen sich zu registrieren und welche motivieren die App auch über einen längerfristigen Zeitraum zu nutzen. Hier ist außerdem die Frage offen, inwiefern es sinnvoll ist die mobile App für den Helfer um Mehrwertfunktion, wie Austauschforen, Belohnungssysteme, Newsletter, etc. zu erweitern. Zur Technikakzeptanz mobiler Systeme siehe etwa (Verkasalo et al. 2010). 
Ebenso sollen Disponenten befragt werden, was sie dazu motivieren würde das AHA-System zu nutzen und wie sie sich eine Integration vorstellen würden, so dass diese ihren Anforderungen genügt und insbesondere in den stressigen Situationen eines Großschadensereignisses effizient und sicher genutzt werden kann. $\mathrm{Zu}$ den besonderen Anforderungen sei auf (Hagemann et al. 2011) verwiesen.

Benutzerschnittstellen auf Seite des freiwilligen Helfers: $\mathrm{Zu}$ betrachten sind die Phasen der Registrierung und der regelmäßigen Nutzung. Während erstere über eine Webseite erfolgt, werden die Einsatz-relevanten Informationen über eine mobile App kommuniziert. Diese soll die Bereitschaft und Position übermitteln. Die Herausforderung besteht hier darin, eine gut bedienbare Oberfläche zur Definition individueller Bereitschaftsregeln und zur Kommunikation im Einsatzfall zu definieren. Bereitschaftsregeln sind dabei orts-, zeit- oder terminabhängig.

Benutzerschnittstelle auf Seiten des Disponenten: Diese muss sich zum einen nahtlos in die gewohnte Umgebung der Leitstelle eingliedern, darf andererseits jedoch keine bestehenden Schwachpunkte übernehmen, hier muss eine detaillierte Usability-Untersuchung erfolgen. Die Anzahl der zur Verfügung gestellten Helfer muss überschaubar sein, um den Disponenten nicht zu überfordern, ihm aber gleichzeitig eine angemessen große Auswahl geben. Die Visualisierung jener Auswahl kann entweder als Liste oder als interaktive Karte erfolgen. Hier ist ebenfalls zu untersuchen, welche Darstellung unter Stress besser zu bedienen ist, denn es ist zu bedenken, dass hier in schwierigsten Situationen zusätzliche Aufmerksamkeit gefordert wird. Human Factors Fragestellungen sind dabei Bestandteil des AHAProjektes, ziehen aber auch bisherige Arbeiten in Betracht, etwa (Danielsson \& Alm 2012).

Usability Tests: Das Testen von Benutzerschnittstellen für Systeme zum Einsatz in Krisensituationen stellt eine besondere Herausforderung dar (Nestler 2014). Für das AHA-Projekt sind geeignete Methoden sowohl für die Seite der freiwilligen Helfer als auch der Disponenten $\mathrm{zu}$ finden und umzusetzen.

\section{Zusammenfassung und Ausblick}

Durch das AHA-Projekt sollen verschiedene Ansätze zur Unterstützung von Rettungskräften kombiniert und in ein bestehendes Leitstellensystem integriert werden. Ziel ist es den Disponenten zu entlasten. Zum Einsatz kommen soll das System vor allem, wenn die regulären Ressourcen knapp werden. Mögliche Einsatzszenarien sind medizinische Hilfeleistungen durch Freiwillige in der direkten Unfallumgebung, technische Hilfeleistung durch das Bereitstellen von Werkzeugen und Fahrzeugen oder das „Erkunden“, d.h. die Überprüfung von Unfalllagen, um ggf. Leerfahrten zu vermeiden. Bis zur Fertigstellung eines Demonstrators sind vor allem noch Fragen der Rechtssicherheit von Kommunen und Helfern, der persönlichen Rechte der Helfer und deren Motivation zu klären. Auf technischer Seite müssen Untersuchungen zur Ortungsqualität und zum Energieverbrauch durchgeführt werden. Außerdem muss das Design der Benutzerschnittstellen für Helfer und Disponent entwickelt werden unter Berücksichtigung von Usability-Kriterien und Tests in den möglichen Einsatzszenari- 
en. Dabei ist in der Leitstelle zu beachten, dass die zusätzliche Arbeitsbelastung möglichst gering ausfällt. Die freiwilligen Helfer müssen über einen längeren Zeitraum motiviert werden und mittels einer App im Einsatzfall gut informiert werden, aber auch bequem, idealerweise automatisiert, ihren Bereitschaftsstatus wechseln können.

\section{Danksagung}

Das Forschungsprojekt AHA wird im Rahmen des Programmes „Forschung für die zivile Sicherheit, Bekanntmachung Zivile Sicherheit - Schutz und Rettung bei komplexen Einsatzlagen“ durch das Bundesministerium für Bildung und Forschung (BMBF) unter dem Kennzeichen 13N13220 gefördert.

\section{Literaturverzeichnis}

Chan, T., Braitberg, G., Elbaum, D., \& Taylor, D. M. (2007). Hatzolah emergency medical responder service: to save a life. Medical journal of Australia, 186(12), 639.

Danielsson, M., \& Alm, H. (2012). Usability and decision support systems in emergency management. Work: A Journal of Prevention, Assessment and Rehabilitation, 41, 3455-3458.

Hagemann, V., Kluge, A., \& Ritzmann, S. (2011). High Responsibility Teams-Eine systematische Analyse von Teamarbeitskontexten für einen effektiven Kompetenzerwerb. Journal Psychologie des Alltagshandelns, 4(1), 22-42.

Hofmann, M., Betke, H., \& Sackmann, S. (2014). Hands2Help-Ein App-basiertes Konzept zur Koordination Freiwilliger Helfer. Themenschwerpunkt: Krisenmanagement Gastherausgeber: Volkmar Pipek und Christian Reuter, 13(1), 36.

Kaufhold, M. A., \& Reuter, C. (2014). Vernetzte Selbsthilfe in Sozialen Medien am Beispiel des Hochwassers 2013. Themenschwerpunkt: Krisenmanagement Gastherausgeber: Volkmar Pipek und Christian Reuter, 13(1), 26.

Nestler, S. (2014). Evaluation der Mensch-Computer-Interaktion in Krisenszenarien. i-com, 13(1), 5362.

Reuter, C., Ludwig, T., \& Pipek, V. (2014). Ad hoc participation in situation assessment: Supporting mobile collaboration in emergencies. ACM Transactions on Computer-Human Interaction (TOCHI), 21(5), 26.

Verkasalo, H., López-Nicolás, C., Molina-Castillo, F. J., \& Bouwman, H. (2010). Analysis of users and non-users of smartphone applications. Telematics and Informatics, 27(3), 242-255.

\section{Kontaktinformationen}

Henrik Detjen, henrik.detjen@hs-ruhrwest.de

Stefan Geisler, stefan.geisler@hs-ruhrwest.de

Gerd Bumiller, gerd.bumiller@hs-ruhrwest.de 